\title{
Evaluation of the Anoxomat: a new technique for anaerobic and microaerophilic clinical bacteriology
}

\author{
J S BRAZIER, S A SMITH The Anaerobe Reference Unit and Department of Clinical Bacteriology, \\ Public Health Laboratory, Luton and Dunstable Hospital
}

SUMMARY A system of automatic jar evacuation-replacement (Anoxomat) for the culture of anaerobes, capnophiles, and microaerophiles was compared with existing methods of anaerobic cabinets, carbon dioxide incubators, and manual evacuation-replacement. Of the 50 species of anaerobes, 29 strains of capnophiles, and 11 strains of microaerophiles tested, equivalent growth was obtained in all but two instances. The Anoxomat system yielded slightly larger colonies in $26(52 \%)$ of anaerobes tested with superior growth in the anerobic cabinets in three $(6 \%)$ of cases and equal in both in $21(42 \%)$. Of the microaerophiles and capnophiles tested, there was no significant difference between the Anoxomat and the conventional system. The Anoxomat system seems to be a suitable alternative to anaerobic and carbon dioxide incubators.

The isolation of certain clinically important organisms that require special atmospheric conditions for growth in vitro places an onus on diagnostic bacteriology laboratories to provide suitable conditions for incubation. Capnophilic pathogens such as Neisseria gonorrhoeae require raised concentrations of carbon dioxide, microaerophiles such as Campylobacter sp require reduced concentrations of oxygen, and obligate anaerobes require no oxygen at all. To meet these demands most modern laboratories use a range of incubators in which the atmospheric conditions are fixed or variable. Alternatively, jars with internal gas generating sachets may be used, or the air may be evacuated by means of a pump and replaced with gas from a cylinder.'

The "Anoxomat" (Mart BV Lichtenvoorde Netherlands, UK agents Jencons Ltd, Leighton Buzzard, Bedfordshire) is designed for use with a cylinder of mixed gases and a pump for jar evacuation to provide gaseous atmospheric conditions for anaerobes, capnophiles, and microaerophiles. The system (figure) is designed to evacuate automatically the air from up to a maximum of three jars at one time and to replace it with a gas mixture suitable for the isolation of microaerophiles, capnophiles, or anaerobes depending on the cycle selected. Such a

Accepted for publication 19 January 1989 system obviates the need for separate anaerobic and microaerophilic incubators, the need to purchase different types of gas kits, and also the need for a supply of pure carbon dioxide.

This report describes "hands on" evaluation of the performance of the Anoxomat for the growth of obligate anaerobes, capnophiles, and microaerophiles and compares it with the existing methods used in the Anaerobe Reference Unit and the Department of Clinical Bacteriology at the Public Health Laboratory, Luton and Dunstable Hospital.

\section{Material and methods}

\section{THE ANOXOMAT CYCLES}

To set up a jar for either anaerobic or microaerophilic conditions the jar is attached to the Anoxomat by a lead which forms a gas tight fit by means of a snap-shut adaptor on the lid. The jar is then evacuated by the selected method after which an audible signal is given to indicate completion of the cycle.

MICROAEROPHILIC CYCLE

The microaerophilic cycle of the Anoxomat was set for a single evacuation of the anaerobic jar at the -0.7 bar and to replace with a gas mixture from a cylinder of compressed gas. A mixture of $80 \%$ nitrogen, $10 \%$ hydrogen, 10\% carbon dioxide (Cryoservice Ltd, Worcester) was used in the trial. The sachet of de-oxo 


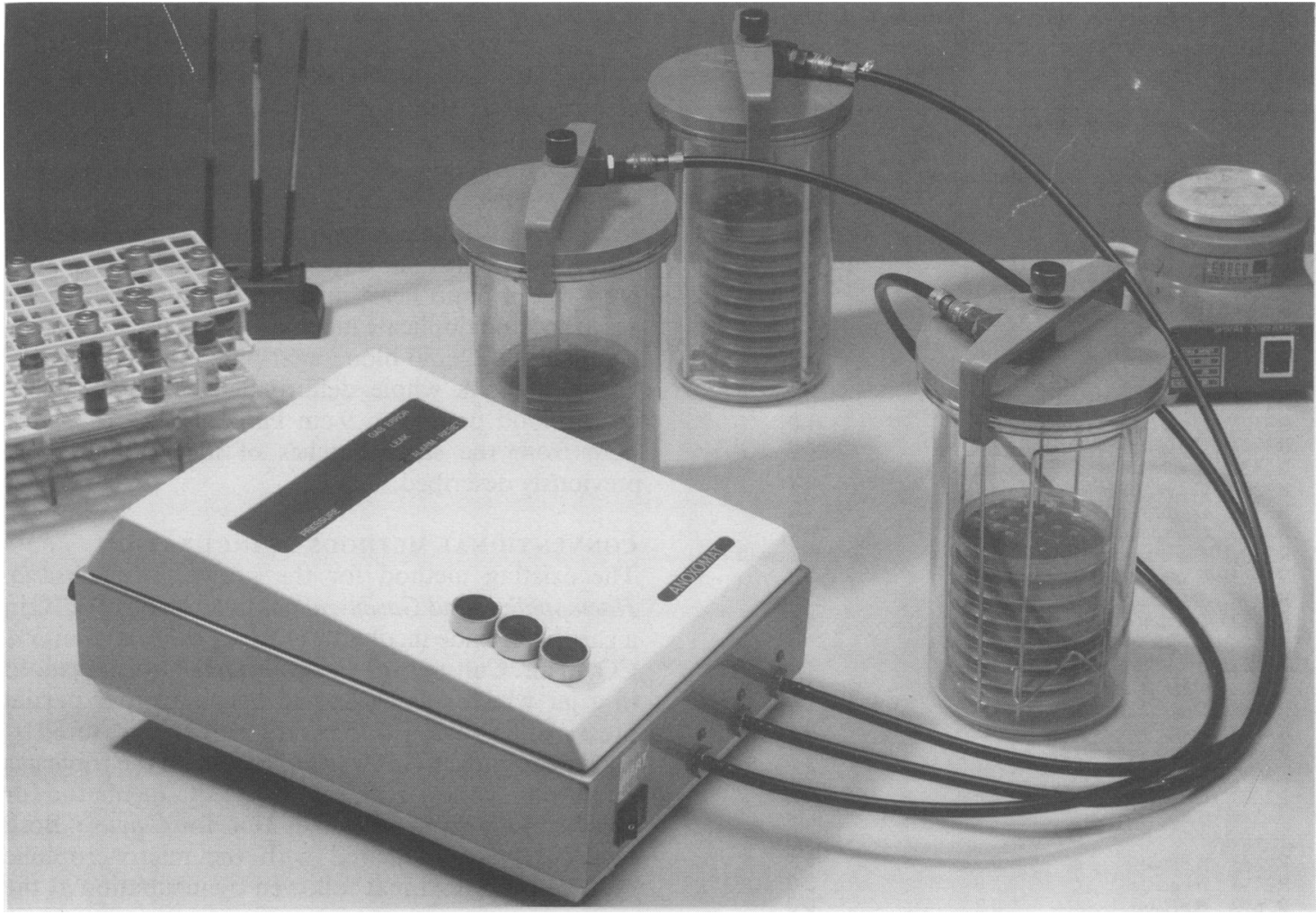

Figure Anoxomat set up for jar evacuation.

palladium catalyst was removed from inside the lid of the anaerobic jar.

\section{ANAEROBIC CYCLE}

The anaerobic cycle evacuates the anaerobic jar at a preselected number of times (range 1-9) to $-0.8 \mathrm{bar}$ and replaces with the gas mixture to a positive pressure of a +0.03 bar. A cycle of three evacuations was chosen with a total cycle time of about 70 seconds. With the catalyst sachet in position any residual oxygen is removed by catalysis.

\section{ANAEROBIC CABINETS}

Two types of anaerobic cabinets were used throughout the study. A Don Whitley Mark II (Don Whitley Scientific Ltd, Shipley) and a Microflow Anaerobic System (MDH Ltd, Andover).

\section{REDOX INDICATORS}

Buffered resazurin indicator $(20 / \mathrm{ml})(0 \cdot 1 \% \mathrm{w} / \mathrm{v})$ freshly decanted from a stock bottle was incubated alongside the culture plates to give an indication of the degree of anaerobiosis achieved.
TEST ORGANISMS AND CULTURE MEDIA

Anaerobes

A range of obligate anaerobes representing the genera Clostridium, Bacteriodes, Peptostreptococcus and Veillonella was chosen from the culture collection of the Anaerobe Reference Unit (table 1). The stock strains had been frozen at $-70^{\circ} \mathrm{C}$ as heavy suspensions in brain heart infusion broth (Oxoid), incorporating $10 \%$ glycerol as the cryo-preservative agent. The suspension was allowed to thaw to room temperature and mixed thoroughly on a vortex mixer, and $10 \mu \mathrm{l}$ was inoculated on to two plates of fastidious anaerobe agar (Lab M Ltd) incorporating 6\% defibrinated horse blood. All plates were poured from the same batch of media by an automatic plate pouring machine (New Brunswick Scientific Ltd) into $9 \mathrm{~cm}$ sterile disposable Petri dishes. All plates were poured to the same depth and dried to the same degree. The inoculum was carefully streaked for single colonies on each plate before incubation. One plate of each pair was placed in an anaerobic cabinet at $37^{\circ} \mathrm{C}$ and the other in the Anoxomat jar. This jar was attached to the Anoxomat and the anaerobic cycle completed. The jar 
Table 1 Comparison of mean colony sizes of anaerobic organisms after 24 hours' incubation in Anoxomat jar and anaerobic cabinets

\begin{tabular}{|c|c|c|c|}
\hline $\begin{array}{l}\text { Reference } \\
\text { No }\end{array}$ & Organism & $\begin{array}{l}\text { Mean colony } \\
\text { size (mm) in } \\
\text { Anoxomat jar }\end{array}$ & $\begin{array}{l}\text { Mean colony } \\
\text { size (mm ) in } \\
\text { anaerobic cabinet }\end{array}$ \\
\hline $\begin{array}{l}\text { Clostridia: } \\
\text { R3283 } \\
\text { R3277 } \\
\text { R3187 } \\
\text { R3088 } \\
\text { R3268 } \\
\text { R2377 } \\
\text { R2302 } \\
\text { R2794 } \\
\text { R3302 } \\
\text { R3267 } \\
\text { R2327 } \\
\text { R3288 } \\
\text { R3318 } \\
\text { R3055 } \\
\text { R2727 } \\
\text { R3303 } \\
\text { R2296 } \\
\text { R2352 }\end{array}$ & $\begin{array}{l}\text { C bifermentans } \\
\text { C botulinum } \\
\text { C butyricum } \\
\text { C cadaveris } \\
\text { C chauvoei } \\
\text { C difficile } \\
\text { C fallax } \\
\text { C novyi A } \\
\text { C paraputrificum } \\
\text { C perfringens } \\
\text { C putrificum } \\
\text { C ramosum } \\
\text { C septicum } \\
\text { C sordellii } \\
\text { C spiroforme } \\
\text { C sporogenes } \\
\text { C tertium } \\
\text { C tetani }\end{array}$ & $\begin{array}{r}1.5 \\
2.0 \\
2.0 \\
2.5 \\
1.5 \\
1.2 \\
2.0 \\
>5.0 \\
1.0 \\
3.0 \\
2.6 \\
0.5 \\
3.0 \\
0.2 \\
0.1 \\
>5.0 \\
1.6 \\
7.5 *\end{array}$ & $\begin{array}{l}1.5 \\
1.0 \\
1.5 \\
2.0 \\
1.0 \\
1.2 \\
1.8 \\
0.5 \\
1.0 \\
3.0 \\
2.0 \\
0.5 \\
4.0 \\
0.2 \\
\text { No growth } \\
>5.0 \\
1.6 \\
4.5\end{array}$ \\
\hline $\begin{array}{l}\text { Fusobacter } \\
\text { R3122 } \\
\text { R2836 } \\
\text { R3163 } \\
\text { R2966 } \\
\text { R3027 } \\
\text { R2803 }\end{array}$ & $\begin{array}{l}\text { ria: } \\
\text { F monidiaformans } \\
\text { F mortiferum } \\
\text { F necrophorum } \\
\text { F nucelatum } \\
\text { F naviforme } \\
\text { F russii }\end{array}$ & $\begin{array}{l}1 \cdot 5 \\
1 \cdot 5 \\
0 \cdot 8 \\
2.0 \\
0 \cdot 1 \\
0.6\end{array}$ & $\begin{array}{l}1 \cdot 0 \\
1 \cdot 0 \\
0 \cdot 3 \\
1 \cdot 5 \\
0 \cdot 1 \\
0 \cdot 2\end{array}$ \\
\hline $\begin{array}{l}\text { Bacteriode } \\
\text { R2863 } \\
\text { R2736 } \\
\text { R2629 } \\
\text { R2514 } \\
\text { R2841 } \\
\text { R2699 } \\
\text { R3054 } \\
\text { R3176 } \\
\text { R2735 } \\
\text { R2840 } \\
\text { R3239 } \\
\text { R2734 } \\
\text { R2314 } \\
\text { R2567 } \\
\text { R2656 }\end{array}$ & $\begin{array}{l}\text { es: } \\
\text { B bivius } \\
\text { B caccae } \\
\text { B disiens } \\
\text { B distasonis } \\
\text { B eggerthii } \\
\text { B fragilis } \\
\text { B hypermegas } \\
\text { B melaninogenicus } \\
\text { B merdae } \\
\text { B ovatus } \\
\text { B praecutus } \\
\text { B stercoris } \\
\text { B thetaiotaomicron } \\
\text { B ureolyticus } \\
\text { B vulgatus }\end{array}$ & $\begin{array}{l}0.3 \\
2.0 \\
0.2 \\
0.8 \\
0.7 \\
1.0 \\
1.3 \\
0.4 \\
0.5 \\
1.1 \\
0.2 \\
0.3 \\
0.9 \\
0.5 \\
1.4\end{array}$ & $\begin{array}{l}0.5 \\
2.0 \\
0.2 \\
0.7 \\
0.3 \\
0.8 \\
1.7 \\
0.3 \\
0.3 \\
0.6 \\
0.1 \\
0.3 \\
0.7 \\
0.4 \\
1.0\end{array}$ \\
\hline $\begin{array}{c}\text { Peptostrep } \\
\text { R3064 } \\
\text { R3330 } \\
\text { R2982 } \\
\text { R3234 } \\
\text { R3238 }\end{array}$ & $\begin{array}{l}\text { ptococci: } \\
P \text { anaerobius } \\
P \text { assacharolyticus } \\
P \text { magnus } \\
P \text { micros } \\
P \text { prevotii }\end{array}$ & $\begin{array}{l}0.5 \\
0.4 \\
0.1 \\
0.1 \\
0.3\end{array}$ & $\begin{array}{l}0.5 \\
0.4 \\
0.1 \\
0.1 \\
0.2\end{array}$ \\
\hline $\begin{array}{c}\text { Veillonella } \\
\text { R2561 } \\
\text { R2649 } \\
\text { R2828 } \\
\text { R2560 } \\
\text { R2648 } \\
\text { R2647 }\end{array}$ & $\begin{array}{l}\text { V atypica } \\
V \text { caviae } \\
V \text { criceti } \\
V \text { dispar } \\
V \text { ratti } \\
V \text { rodentium }\end{array}$ & $\begin{array}{l}0.2 \\
0.4 \\
0 \cdot 5 \\
0 \cdot 4 \\
0.5 \\
0.3\end{array}$ & $\begin{array}{l}0.2 \\
0.4 \\
0.5 \\
0.3 \\
0.5 \\
0.3\end{array}$ \\
\hline
\end{tabular}

* Radius of swarm.

†Mean diameter of five representative colonies.

was incubated in a $37^{\circ} \mathrm{C}$ incubator. Measurements were taken to ensure that temperatures for both incubation systems were identical.
Microaerophiles and capnophiles

A range of microaerophilic and capnophilic organisms isolated from clinical specimens submitted to the diagnostic bacteriology laboratory at the Luton and Dunstable Hospital was chosen. These included; Gardnerella vaginalis (10 strains), $N$ gonorrhoeae (10 strains), Haemophilus influenzae (6 strains), $C$ jejuni/ coli (10 strains), Streptococcus milleri (3 strains), and $C$ pylori (1 strain). The inoculum of each strain was prepared in brain heart infusion broth as previously described and duplicate inocula of $10 \mu \mathrm{l}$ were made on two plates of Oxoid blood agar base No 2 incorporating either $6 \%$ whole defibrinated or chocolatised horse blood poured in $9 \mathrm{~cm}$ Petri dishes. All plates were from the same batches of media poured as previously described.

\section{CONVENTIONAL METHODS OF INCUBATION}

The existing method for the growth of Neisseria, Haemophilus, and Gardnerella is incubation at $37^{\circ} \mathrm{C}$ in a carbon dioxide incubator (LEEC) calibrated at $5 \%$ $\mathrm{CO}_{2}$ in air. Cultures for Campylobacter sp were placed in a jar which was evacuated by pump to a partial pressure of $57 \mathrm{~cm}(-0.75$ bar) of mercury measured by a pressure gauge. This was replaced with the triple gas mixture and the jar placed in a $43^{\circ} \mathrm{C}$ incubator for growth of $C$ jejuni/coli, and at $37^{\circ} \mathrm{C}$ for $C$ pylori. Both methods were compared with the microaerophilic cycle of the Anoxomat followed by incubation at the appropriate temperature.

Each set of plates was examined after 24 and 48 hours' incubation. Five representative colonies of each strain were measured and the mean diameter of each strain calculated. From these figures an overall mean for each organism was calculated. Colony diameters were measured using a plate microscope and an eyepiece graticule calibrated in 0.5 millimetre divisions. Colonies were illuminated by an external fibre optic light source. The total number of colonies on each plate was compared by the $+1-$ to +++ notation.

For the microaerophilic and capnophilic organisms where more than one strain of each species was tested, the five individual readings of colony sizes of each strain compared under the different conditions of incubation were used to calculate the standard deviation for each organism. From these figures, together with the mean colony sizes for each strain, the standard error of difference between two means was established using the equation:

Standard error of difference between means $=$

$$
\sqrt{\frac{\left(\mathrm{SD}_{1}\right)^{2}}{\mathrm{n}}+\frac{\left(\mathrm{SD}_{2}\right)^{2}}{\mathrm{n}}}
$$

( $n=$ number of strains tested).

The figure generated permits the calculation of how 
many multiples of the difference between the means this figure represents, and by reference to a probability table ${ }^{2}$ facilitates a statistical comparison of the results. For the anaerobic organisms and microaerophiles where only a single strain of each species was used statistical analysis was not applied.

\section{Results}

The results of the comparisons of colony sizes of the anaerobes after 24 hours' incubation among the various incubation systems are listed in table 1 . All plates except one yielded +++ growth. Clostridium spiroforme had not grown in the anaerobic cabinet at 24 hours, but in the Anoxomat jar a + growth was visible. There were no other differences in the total amount of growth between the two anaerobic systems. Of the anaerobic organisms tested, $26(52 \%)$ of test organism colonies were larger (range $0.1-1.0 \mathrm{~mm}$ ) on the Anoxomat plates at 24 hours than on those incubated in either of the anaerobic cabinets. Six per cent were larger (range $0.1-0.2 \mathrm{~mm}$ ) in the anaerobic cabinets, and the remaining $21(42 \%)$ were equal in both. At 48 hours the difference was less noticeable with $16(33 \%)$ of colonies in the Anoxomat jar larger by $0 \cdot 1-0.2 \mathrm{~mm}$ and the remaining $34(67 \%)$ being equal in both. The resazurin indicator generally became decolourised faster in the Anoxomat jar than in either of the anaerobic cabinets.

The results of the microaerophilic and capnophilic strains tested after 48 hours' incubation are listed in table 2. While there were some measurable differences in colony sizes, these did not prove significant $(p<$ 0.317 ). One strain of $C$ jejuni/coli did not grow in the conventional jar system but grew in the Anoxomat jar.

\section{Discussion}

The Anoxomat is essentially an automated system for the evacuation and gas replacement of jars used for microaerophilic or anaerobic atmospheric conditions.
The fact that the results obtained with the anaerobic test organisms were superior in $52 \%$ of cases with the Anoxomat system than the anaerobic cabinets was a surprise because previous workers ${ }^{3-5}$ have reported that anaerobic cabinets yield superior growth to that of a jar system both in internally generated gases or in the single evacuation-replacement method. Berry $e t$ al, however, reported that in a comparison of a prototype of the Gallenkamp anaerobic incubator colonies were slightly smaller in the cabinet compared with those obtained in a jar system, ${ }^{6}$ and Watt $e t$ al showed no quantitative difference between jars and anaerobic cabinets in the recovery of strictly anaerobic clostridia. ${ }^{7}$ As other factors affecting colony size were all standardised the factor which varied between the two systems was the speed by which anaerobiosis was achieved as shown by the differences seen in the decolourisation of the resazurin indicator. This would enable anaerobes to enter a logarithmic phase of growth sooner so that surface colonies would appear larger, as was generally the case in the present study after 24 hours' incubation.

The Anoxomat was very easy to use: the swapping of pump and manometer leads was eliminated as was surveillance for secondary vacuum production normally associated with single evacuation replacement. The lack of evidence of catalytic activity, however, may be regarded as a disadvantage in the control of the system because there is no other indication that the catalyst is functioning and no redox indicators are supplied with the system. Because the jar is partially evacuated three times there is less residual oxygen for removal by catalysis; nevertheless, the inclusion of an indicator such as resazurin is to be recommended to give the user some indication of the degree of catalyst activity. Alternatively, a control organism such as $C$ tetani could be used.

The results of the comparisons using capnophilic organisms indicate that the Anoxomat microaerophilic cycle would be adequate for the growth of Neisseria, Haemophilus, and Gardnerella.

Table 2 Comparison of mean colony sizes of microaerophiles and capnophiles after 48 hours' incubation in Anoxomat and conventional systems

\begin{tabular}{|c|c|c|c|c|c|c|c|}
\hline \multirow[b]{3}{*}{ Organisms } & \multirow[b]{3}{*}{ No } & \multicolumn{6}{|c|}{ Mean colony sizes ( $\mathrm{mm}$ ) at 48 hours } \\
\hline & & \multicolumn{2}{|c|}{ Anoxomat } & \multicolumn{2}{|c|}{ Conventional } & \multirow[b]{2}{*}{$S E$} & \multirow[b]{2}{*}{ p Value* } \\
\hline & & Mean & $(S D)$ & Mean & $(S D)$ & & \\
\hline $\begin{array}{l}N \text { gonorrhoeae } \\
\text { Campylobacter sp } \\
\text { Gardnerella sp } \\
\text { Haemophilus sp } \\
\text { Streptococcus milleri } \\
\text { C pylori }\end{array}$ & $\begin{array}{r}10 \\
10 \\
10 \\
6 \\
3 \\
1\end{array}$ & $\begin{array}{l}0.82 \\
0.52 \\
0.36 \\
1.38 \\
0.83 \\
0.46\end{array}$ & $\begin{array}{l}(0.1316) \\
(0.475) \\
(0.107) \\
(0 \cdot 194) \\
(0.351)\end{array}$ & $\begin{array}{l}0.93 \\
0.42 \\
0.33 \\
1.53 \\
0.83 \\
0.42\end{array}$ & $\begin{array}{l}(0.323) \\
(0.248) \\
(0.074) \\
(0.372) \\
(0.231)\end{array}$ & $\begin{array}{l}1.000 \\
1.275 \\
1.360 \\
1.731 \\
0.440\end{array}$ & $\begin{array}{r}0.317 \\
<0.317 \\
<0.317 \\
<0.100 \\
>0.500\end{array}$ \\
\hline
\end{tabular}

*Probability of observation showing at least as large a deviation from the population mean. 
As to cost effectiveness, the Anoxomat system retains at around $£ 3500$, which is about the same price as some of the cheaper anaerobic cabinets on the market, so at first it might seem an expensive alternative, bearing in mind the additional cost of the anaerobic jars. For a laboratory using this system to culture microaerophiles, however, as well as anaerobes, the saving on carbon dioxide incubators, gas generating kits, and pure carbon dioxide gas supplies would make the costs comparable. No direct comparison was made with internal gas generated kits. Modern laboratories will no doubt be loth to dispense with their existing incubators for culturing anaerobes and microaerophiles but for small laboratories or laboratories in countries where maintenance of anaerobic cabinets proves difficult the Anoxomat may be suitable.

The arguments about the relative merits of anaerobic jars and anaerobic cabinets still stand. Anaerobic cabinets provide the bonus that plates can be examined without disturbance of anaerobiosis and permit manipulation of specimens in continuous anaerobic conditions. The Anoxomat jar system scores in its versatility because it can be used to grow the full range of non-aerobic clinically important organisms. This versatility is a strong point in favour of the Anoxomat for those laboratories who prefer to use jars rather than a range of incubators.

We thank Mrs J Hooker for her technical assistance and Dr AT Willis for his support.

\section{References}

1 Fildes P, McIntosh J. An improved form of anaerobic jar. Br J Exp Pathol 1921;2:153.

2 Armitage P. Probability related to multiples of standard deviations for a normal distribution. In: Swinscow TDV, ed. Statistics at square one. London: BMA, 1985:79.

3 Rosebury T, Reynolds JB. Continuous anaerobiosis for cultivation of spirochaetes. Proc Soc Exp Biol Med 1964;117:813-5.

4 Drasar BS. Cultivation of anaerobic intestinal bacteria.J Pathol Bacteriol 1967;94:417-27.

5 Aranki A, Syed SA, Kenney EB, Freter R. Isolation of anaerobic bacteria from human gingiva and mouse cecum by means of a simplified glove box procedure. Appl Microbiol 1969;17:568-76.

6 Berry PL, Taylor E, Phillips I. The use of an anaerobic incubator for the isolation of anaerobes from clinical samples. $J$ Clin Pathol 1982;35:1158-62.

7 Watt B, Collee JG, Brown R. The isolation of strict anaerobes: The use of an anaerobic cabinet compared with a conventional procedure. J Med Microbiol 1974;7:315-24.

Requests for reprints to: J S Brazier, Anaerobe Reference Unit, Public Health Laboratory, Luton and Dunstable Hospital, Lewsey Road, Luton LU4 0DZ, England. 